\title{
Large-Time Behavior of Discrete Velocity Boltzmann Equations
}

\author{
J. Thomas Beale ${ }^{1}$ \\ Department of Mathematics, Duke University, Durham, NC 27706, USA
}

\begin{abstract}
We study the asymptotic behavior of equations representing onedimensional motions in a fictitious gas with a discrete set of velocities. The solutions considered have finite mass but arbitrary amplitude. With certain assumptions, every solution approaches a state in which each component is a traveling wave without interaction. The techniques are similar to those in an earlier treatment of the Broadwell model [1].
\end{abstract}

\section{Introduction}

The purpose of this paper is to describe the evolution at large times of solutions to certain systems of semilinear hyperbolic equations. These equations represent onedimensional motions in a fictitious gas consisting of particles with a discrete set of possible velocities. The study of such model gases began with Maxwell. The systems studied here have the form

$$
u_{i, t}+c_{i} u_{i, x}=\sum_{j, k=1}^{n} a_{i}^{j k} u_{j} u_{k} \equiv F_{i}, \quad i=1, \ldots, n,
$$

where $u_{i}(x, t)$ is the density of particles with speed $c_{i}$, and the interaction coefficients $a_{i}^{j k}$, as well as the speeds, are constant. We suppose that the speeds are distinct:

$$
c_{i} \neq c_{j} \quad \text { if } \quad i \neq j
$$

With certain assumptions on the system, we show that each solution of finite total mass, but arbitrary amplitude, evolves toward a state in which the interaction is negligible; i.e., there are limiting wave forms $u_{i}^{\infty}$ so that

$$
u_{i}(x, t) \sim u_{i}^{\infty}\left(x-c_{i} t\right) \quad \text { as } \quad t \rightarrow \infty, \quad i=1, \ldots, n .
$$

\footnotetext{
${ }^{1}$ Partially supported by N.S.F. Grant No. NSF-DMS-84-08393
} 
If for some $i$ the $i$-equation contains a decay term proportional to $-u_{i}^{2}$, then $u_{i}^{\infty}=0$. Thus the exchange of mass through the nonlinear terms may cause local growth in some components for intermediate times, but the ultimate character of the solution is determined by the transport at different speeds. Results of this type have been derived for general systems of equations for solutions of small amplitude by Tartar [14] and for the three-component Broadwell system with arbitrary amplitude by the author [1]. The Broadwell system is a primary example of the class of equations considered here.

We will make several assumptions on the system (1.1). The Boltzmann-type equations for a multi-dimensional discrete velocity gas reduce in the case of onedimensional motions to a system satisfying these assumptions, provided certain natural conditions are satisfied by the collisions in the model gas. This reduction is discussed in detail in Sect. 2. First, we assume sign conditions on the interaction coefficients:

$$
\begin{aligned}
& a_{i}^{j k} \geqq 0 \quad \text { if } \quad j \neq i \text { and } k \neq i \text {, } \\
& a_{i}^{j k} \leqq 0 \quad \text { if } \quad j=1 \quad \text { or } \quad k=i \text {. }
\end{aligned}
$$

Thus $i$-particles may only be created in an interaction in which they do not enter, and otherwise, they may only be lost. We may as well suppose the coefficients are symmetric in $j, k$ :

$$
a_{i}^{j k}=a_{i}^{k j} .
$$

We will assume that mass is conserved in the individual interactions, so that for each $j, k$, the sum of coefficients of terms $u_{j} u_{k}$ is zero. We allow weight coefficients $v_{i}>0$; they arise in identifying different particles with speed $c_{i}$, as explained in Sect. 2. Thus with fixed constants $v_{i}$ our conservation of mass condition is

$$
\sum_{i=1}^{n} v_{i} a_{i}^{j k}=0, \quad j, k=1, \ldots, n .
$$

We also assume conservation of momentum:

$$
\sum_{i=1}^{n} v_{i} c_{i} a_{i}^{j k}=0, \quad j, k=1, \ldots, n
$$

We further assume an entropy condition, which is a consequence of reversibility of the collisions:

$$
\sum_{i} v_{i} F_{i} \log u_{i} \leqq 0 \quad \text { when } \quad u_{1}, \ldots, u_{n}>0
$$

Finally we will need a more technical condition concerning the occurrence of terms $u_{i}^{2}$, which will always be satisfied for the gas models described in Sect. 2 . We note that for given $i$, either no square terms occur, i.e., $a_{j}^{i i}=0$ for all $j$; or otherwise $a_{i}^{i i}<0$, because of the sign conditions and conservation of mass. Our assumption is

$$
\begin{aligned}
& \text { If } a_{i}^{i i}<0 \text {, then there is a sequence of indices } i=i_{1}, i_{2}, \ldots, i_{r}, \\
& r \geqq 2 \text {, so that for } k=1, \ldots, r-1, a_{i_{k+1}}^{i_{k} i_{k}}>0 \text {, and } a_{i_{r}}^{i_{r} i_{r}}=0 .
\end{aligned}
$$

The main results of this paper are summarized in the following theorems. It is supposed that $u(x, t)$ is a solution of the system (1.1) with conditions (1.2)-(1.9) 
satisfied. We assume initial condition $u_{i}(x, 0)=u_{0 i}(x), i=1, \ldots, n$, with $u_{0 i} \geqq 0$ prescribed in $L^{1} \cap L^{\infty}$. The solution is in the class $C\left(0, T ; L^{p}(\mathbb{R})\right)$ for $1 \leqq p<\infty$, with $T>0$ arbitrary; the existence theory is discussed in Sect. 3. In particular, discontinuities are allowed.

Theorem 1 ( $\left(\mathrm{L}^{1}\right.$ Asymptotics). There exist functions $u_{i}^{\infty} \in L^{1}(\mathbb{R}), i=1, \ldots, n$ so that $u_{i}\left(\cdot+c_{i} t, t\right) \rightarrow u_{i}^{\infty}$ in $L^{1}(\mathbb{R})$ as $t \rightarrow \infty$. If for some $i, a_{i}^{i i}<0$, then $u_{i}^{\infty}=0$.

Theorem 2 (Boundedness and $L^{p}$ Asymptotics). The solution $u(x, t)$ is uniformly bounded for $x \in \mathbb{R}, t>0$, and each $u_{i}^{\infty}$ is in $L^{\infty}(\mathbb{R})$. The convergence to $u_{i}^{\infty}$ takes place in $L^{p}(\mathbb{R})$ for $1 \leqq p<\infty$.

Theorem 3 (Uniform Asymptotics). Assume that the $u_{0 i}$ tend to zero as $x \rightarrow \pm \infty$. Then $u_{i}^{\infty}$ tends to zero as $x \rightarrow \pm \infty$. The convergence of $u_{i}\left(\cdot+c_{i} t, t\right)$ to $u_{i}^{\infty}$ as $t \rightarrow \infty$ takes place in $L^{\infty}(\mathbb{R})$. If the initial state is $C^{1}$, then $u_{i}^{\infty}$ is continuous.

The solution is nonnegative provided the initial data are. Because of (1.6), the total mass

$$
\int_{-\infty}^{\infty} \sum_{i=1}^{n} v_{i} u_{i}(x, t) d x \equiv m
$$

is conserved in time, and similarly (1.7) implies the conservation of total momentum

$$
\int_{-\infty}^{\infty} \sum_{i=1}^{n} v_{i} c_{i} u_{i}(x, t) d x
$$

Equation (1.10) means that the $L^{1}$ norm is constant, but it seems that no natural higher norm is nonincreasing. For this reason the approach to describing the large-time behavior must be somewhat indirect. The method used here is similar to that in [1] but there are important differences. In fact, one significant aspect of the present treatment is that the asymptotic description holds with only two assumed conservation laws, those of mass and momentum, although the number of components may be large. In contrast to [1], we first derive the asymptotic limit in $L^{1}$ (Theorem 1) without estimates in higher norms. We then use this result to advantage in proving Theorem 2.

The results here include the asymptotic description of the Broadwell system in [1] as a special case, except that we considered only smooth solutions in the earlier work. In the Broadwell case we were able to show that the limiting state depends continuously on the initial data (Theorem 3 of [1]), but we have not been able to show this for general systems. Our approach here uses ideas from Tartar's work [14] and extends the results on asymptotics to arbitrary amplitude, although there are slightly different assumptions on the system (1.1). Other work on systems like (1.1) may be found in [2-13] and in the references listed in [1].

In Sect. 2, we discuss the Boltzmann equation of the model gas and describe conditions leading to one-dimensional solutions governed by the system (1.1) with assumptions (1.2)-(1.9). We then summarize in Sect. 3 the existence theory for (1.1) following an argument of Crandall and Tartar. In Sect. 4 we prove (Lemma 4.1) 
that the interaction terms are integrable in spacetime; as noted in [14], this easily implies the $L^{1}$ convergence of Theorem 1. We use weight functions in the equations analogous to those of [1] for the Broadwell system, but they are used in a different way. In Sect. 5 we use related weight functions to show that the $L^{p}$ norm remains bounded at large time; Theorem 2 follows by letting $p \rightarrow \infty$. Finally in Sect. 5 we derive the uniform convergence to the limiting state with slightly more assumed about the initial data.

It is not apparent whether methods such as these would apply to solutions confined to an interval with appropriate boundary conditions. We would not expect the interaction terms to be integrable in space-time in this case. Except for the Carleman model, asymptotic results for large data have not been given. In higher space dimensions there are no existence results for all time except for small data. The weight functions used here involve one-dimensional integrals which are automatically bounded by the total mass; this would not be the case in more than one space dimension. R. Illner has shown that for the two-dimensional Broadwell system, solutions of finite mass may grow indefinitely in maximum norm. The description of solutions in higher dimensions will therefore have to be somewhat different from that of the one-dimensional case treated here.

\section{The Discrete Velocity Boltzmann Equation}

We now describe the equations of the discrete velocity gas and verify that onedimensional motions reduce to the system (1.1) with assumptions (1.2)-(1.9) satisfied. For a fuller discussion of model gases of this type, see the lecture notes by Gatignol [5] and Cabannes [3] and the survey [6].

We suppose that the gas consists of particles of identical mass with vector velocities $V_{1}, \ldots, V_{N}$. A pair of particles with velocities $V_{K}, V_{L}$ may collide and result in a new pair of particles with velocities $V_{I}, V_{J}$ provided momentum and energy are conserved:

$$
\begin{gathered}
V_{I}+V_{J}=V_{K}+V_{L}, \\
\left|V_{I}\right|^{2}+\left|V_{J}\right|^{2}=\left|V_{K}\right|^{2}+\left|V_{L}\right|^{2} .
\end{gathered}
$$

The particles with velocity $V_{I}$ have density $U_{I}(x, y, z, t)$. The amount of mass of each component gained or lost in a volume element $d x d y d z$ in time $d t$ is $A_{I J}^{K L} U_{K} U_{L} d x d y d z d t$, where $A_{I J}^{K L}$ is a nonnegative constant specifying the transition rate of the reaction. If (2.1), (2.2) are not satisfied, then $A_{I J}^{K L}=0$. From the definition the coefficients are symmetric in each pair of indices,

$$
A_{J I}^{K L}=A_{I J}^{K L}=A_{I J}^{L K} \text {. }
$$

We make the assumption that the reverse reaction is equally likely,

$$
A_{K L}^{I J}=A_{I J}^{K L} \text {. }
$$

It is simple to check that if $J=I$, then (2.1), (2.2) can be satisfied only in the trivial case $K=L=I$. Furthermore, if $K=I,(2.1)$ implies $L=J$. Using these remarks, the equations of evolution for the density functions can be written as

$$
U_{I, t}+\left(V_{I} \cdot \nabla\right) U_{I}=\frac{1}{2} \sum_{J, K, L} A_{I J}^{K L}\left(U_{K} U_{L}-U_{I} U_{J}\right) \equiv \Phi_{I}\left(U_{1} \ldots U_{N}\right)
$$


(see Sect. I.A of [5]). For simplicity we will replace $A_{I J}^{I J}=A_{I J}^{J I}$ by zero; this obviously has no effect on the equations.

We consider solutions of (2.5) which are independent of $y, z$. Let $C_{I}=V_{I} \cdot \hat{x}$, where $\hat{x}$ is the unit vector in the $x$-direction. Then (2.5) becomes

$$
U_{I, t}+C_{I} U_{I, x}=\Phi_{I}\left(U_{1}, \ldots, U_{N}\right) .
$$

It is not hard to see that this system satisfies the sign conditions (1.3), (1.4), the mass and momentum conservation (1.6), (1.7), and the entropy condition (1.8); for the last see Sect. I.D. of [5] or Sect. 2.1 of [3]. We wish to reduce this system however, by combining densities $I^{\prime}, I$ for which $C_{I^{\prime}}=C_{I}$, and to do this we need a further structural assumption.

We introduce an equivalence relation on the indices $1, \ldots, N$, viz. $I^{\prime} \sim I$ if $C_{I^{\prime}}=C_{I^{\prime}}$. We will need a symmetry condition for the coefficients in (2.5):

If $I^{\prime} \sim I$, there is a bijection on $\{1, \ldots, N\}$ preserving equivalence classes and taking $I \rightarrow I^{\prime}$ so that for all $J, K, L$ we have $A_{I^{\prime} J^{\prime}}^{K^{\prime} L^{\prime}}=A_{I J}^{K L}$, where $J^{\prime}, K^{\prime}, L^{\prime}$ are the images of $J, K, L$.

A simple consequence of (2.7) will allow us to combine densities in the same equivalence class:

If $U_{1}, \ldots, U_{N}$ have the property that $J^{\prime} \sim J$ implies $U_{J^{\prime}}=U_{J}$, then $I^{\prime} \sim I$ implies $\Phi_{I^{\prime}}\left(U_{1}, \ldots, U_{N}\right)=\Phi_{I}\left(U_{1}, \ldots, U_{N}\right)$.

Next we number the equivalence classes $1, \ldots, n$ in some fashion, $n \leqq N$, and write $i=[I]$ when $I$ is in the equivalence class $i$. Let $v_{i}$ be the number of elements in the $i^{\text {th }}$ class and $c_{i}=C_{I}$ with $i=[I]$. We now consider density functions $u_{1}(x, t), \ldots, u_{n}(x, t)$ and associate $U_{1}, \ldots, U_{N}$ by defining $U_{I}=u_{[I]}$. Also let $F_{i}\left(u_{1}, \ldots, u_{n}\right)=\Phi_{I}\left(u_{[1]}, \ldots, u_{[N]}\right)$, with $I$ any member of class $i$. We now compare the system

$$
u_{i, t}+c_{i} u_{i, x}=F_{i}\left(u_{1}, \ldots, u_{n}\right)
$$

with (2.6). If $u_{1}(x, t), \ldots, u_{n}(x, t)$ solve (2.9), then because of (2.8) the corresponding $U_{I}$, as defined above, satisfy (2.6). As shown by an argument in Sect. 3, the solutions of (2.6) are uniquely determined by initial data. Thus if $U_{01}(x), \ldots, U_{0 N}(x)$ are prescribed data for (2.6) with $U_{0 J^{\prime}}=U_{0 J}$ for $J^{\prime} \sim J$, we may find the solution by solving the reduced system (2.9) with $u_{0[J]}=U_{0 J}$ and then setting $U_{I}=u_{[I]}$.

It remains to check that the system (2.9) satisfies all the conditions (1.2)-(1.9) under our present assumptions. To put (2.9) in the form (1.1), we combine terms in $F_{i}$ as defined above from $\Phi_{I}$. The sum in (2.5) may contain terms with $K \neq I$ but $K \sim I$; then $C_{K}=C_{I}$, and from (2.1), $C_{L}=C_{J}$ as well. Thus $L \sim J$, and $U_{K} U_{L}=U_{I} U_{J}$, so that the $(J, K, L)$-term in the sum is zero. After canceling such terms we have only positive terms $u_{k} u_{l}$ with $k \neq[I]$ and $l \neq[I]$, and negative terms $u_{[I]} u_{j}$. Therefore (2.9) has the form (1.1) with the sign conditions (1.3), (1.4) satisfied. The conservation laws (1.6), (1.7) and the entropy condition (1.8) are direct consequences of those for (2.6).

Finally we verify condition (1.9) for the square terms. Suppose $a_{i}^{i i}<0$ for some $i$. Then for some $I, J, K$ with $[I]=[J]=i,(2.1)$ is satisfied. With $k=[K], l=[L]$, we have $2 c_{i}=c_{k}+c_{l}$. Since the $c$ 's are distinct, one of $c_{k}, c_{l}$, is strictly greater than $c_{i}$, say $c_{k}$. Set $i_{1}=i, i_{2}=k$. If $a_{i_{2}}^{i_{2} i_{2}}=0$, we set $r=2$, and (1.9) is satisfied. If terms with $u_{i_{2}}^{2}$ 
occur, we repeat the above argument to find $i_{3}$ with $c_{i_{3}}>c_{i_{2}}$. If $a_{i_{3}}^{i_{3} i_{3}}=0$, we stop; otherwise we continue. This process eventually terminates since there are only finitely many speeds. If $c_{i_{r}}$ is the maximal speed, there are no nontrivial choice of $k, l$ so that $2 c_{i_{r}}=c_{k}+c_{l}$, and therefore no terms with $u_{i_{r}}^{2}$ appear.

The Broadwell system, treated in [1], serves as an example of the general development above. In this case the velocity $V_{I}$ is the unit vector in the $I^{\text {th }}$ coordinate direction, $I=1,2,3$, and $V_{I+3}=-V_{I}$. Equations (2.6) are

$$
\begin{gathered}
U_{1, t}+U_{1, x}=U_{2} U_{5}+U_{3} U_{6}-2 U_{1} U_{4}, \\
U_{2, t}=U_{3} U_{6}+U_{1} U_{4}-2 U_{2} U_{5}, \\
U_{3, t}=U_{1} U_{4}+U_{2} U_{5}-2 U_{3} U_{6},
\end{gathered}
$$

with the same right-hand sides for $U_{I+3}$ as for $U_{I}$. Here $I=2,3,5,6$ are combined in one equivalence class, with $U_{1}, U_{4}$ each remaining separate. The condition (2.7) holds for each pair $I, I^{\prime}$ from $\{2,3,5,6\}$. For example, if $I=2, I^{\prime}=5$, the mapping interchanges 2,5 . The case $I=3, I^{\prime}=6$ is analogous. For $I=2, I^{\prime}=3$, we interchange 2,3 and also 5,6. Other cases can be regarded as composites of these. Note that it is not true that $A_{I^{\prime} J^{\prime}}^{K^{\prime}}=A_{I J}^{K L}$ whenever corresponding indices are in the same equivalence class.

\section{Existence Theory}

We now discuss existence and basic properties for the system (1.1). The existence theory needed is essentially that of Tartar [14] but some remarks are necessary because of slightly different hypotheses. Our fundamental result is the following.

Existence Theorem. Suppose the system (1.1) satisfies conditions (1.2)-(1.8), and suppose nonnegative initial data $u_{0 i}, i=1, \ldots, n$, are given in $L^{1} \cap L^{\infty}$. Then there exists a unique nonnegative solution of (1.1) for all time in the class $C\left(0, \infty ; L^{p}(\mathbb{R})\right)$ for $1 \leqq p<\infty$, with the prescribed initial value. There is a function $G\left(T, K_{0}\right)$ so that if $\left|u_{0 i}\right|_{L^{\infty}} \leqq K_{0}$, then $\left|u_{i}(\cdot, t)\right|_{L^{\infty}} \leqq G\left(T, K_{0}\right)$ for $t \leqq T$. If $u_{0}$ is $C^{1}$ in $x$, then $u$ is $C^{1}$ in $(x, t)$.

We outline the proof of this theorem, assuming first that the data are $C^{1}$. A solution can be constructed for short time by writing the equations in integral form along the characteristics and using the contraction mapping principle. The length of time depends only on the maximum of the initial data. A domain of dependence property is evident from this construction: the solution at $\left(x_{0}, t_{0}\right)$ depends only on the initial data on the interval $\left|x-x_{0}\right| \leqq c_{\max } t, c_{\max }=\max _{i}\left|c_{i}\right|$. As noted in [14] it is also evident that nonnegative data lead to a nonnegative solution provided we define the contraction mapping as $u \rightarrow S u$,

$$
(S u)_{i, t}+c_{i}(S u)_{i, x}=\sum_{j, k \neq i} a_{i}^{j k} u_{j} u_{k}+\sum_{j}\left(2-\delta_{i j}\right) a_{i}^{i j}(S u)_{i} u_{j}
$$

Uniqueness is easily checked in the class $C^{1}$. From (1.6), (1.7) we have the laws of mass and momentum conservation:

$$
\begin{gathered}
\sum_{j} v_{j} u_{j, t}+\sum_{j} v_{j} c_{j} u_{j, x}=0, \\
\sum_{j} v_{j} c_{j} u_{j, t}+\sum_{j} v_{j} c_{j}^{2} u_{j, x}=0 .
\end{gathered}
$$


Next we check that solutions of small mass remain uniformly bounded independent of time, using an argument like that of Nishida and Mimura for the Broadwell system. (Cabannes [4] has recently given a boundedness argument for small data related to this one.) Integrating the $i^{\text {th }}$ equation along its characteristic, we have

$$
\begin{aligned}
u_{i}(x, t) & =u_{i_{0}}(x)+\int_{0}^{t} F_{i}\left(x+c_{i}\left(t^{\prime}-t\right), t^{\prime}\right) d t^{\prime} \\
& \leqq u_{i_{0}}(x)+C \sum_{j, k \neq i} \int_{0}^{t}\left(u_{j} u_{k}\right)\left(x+c_{i}\left(t^{\prime}-t\right), t^{\prime}\right) d t^{\prime} .
\end{aligned}
$$

We now apply the divergence theorem to each conservation law (3.1), (3.2) on the region $\left\{\left(x^{\prime}, t^{\prime}\right): x^{\prime}<x+c_{i}\left(t^{\prime}-t\right), 0<t^{\prime}<t\right\}$ to obtain, with $\alpha=0$ or 1 ,

$$
\begin{gathered}
\int_{-\infty}^{x} \sum_{j} v_{j} c_{j}^{\alpha} u_{j}\left(x^{\prime}, t\right) d x^{\prime}-\int_{-\infty}^{x-c_{i} t} \sum_{j} v_{j} c_{j}^{\alpha} u_{0 j}\left(x^{\prime}\right) d x^{\prime} \\
=\int_{0}^{t} \sum_{j} v_{j} c_{j}^{\alpha}\left(c_{j}-c_{i}\right) u_{j}\left(x+c_{i}\left(t^{\prime}-t\right), t^{\prime}\right) d t^{\prime} .
\end{gathered}
$$

Taking the momentum identity minus $c_{i}$ times the mass identity, we get

$$
\sum_{j} \int_{0}^{t} v_{j}\left(c_{j}-c_{i}\right)^{2} u_{j}\left(x+c_{i}\left(t^{\prime}-t\right), t^{\prime}\right) d t^{\prime} \leqq C m,
$$

since the integrals on the left above represent part of the mass. Therefore by (1.2)

$$
\sum_{j \neq i} \int_{0}^{t} u_{j}\left(x+c_{i}\left(t^{\prime}-t\right), t^{\prime}\right) d t^{\prime} \leqq C^{\prime} m .
$$

We use this in (3.3); if $K_{0}$ is the maximum of the initial data, and $K$ the maximum up to time $T$, then $K \leqq K_{0}+C_{0} K m$, where $C_{0}$ is a constant depending only on the coefficients in the equations. Finally, we have $K \leqq K_{0} /\left(1-C_{0} m\right)$ provided $m<C_{0}^{-1}$, a bound independent of the time interval. Thus if $m \leqq 1 / 2 C_{0}$, the shorttime existence argument can be repeated to obtain a solution for all time which is uniformly bounded.

Now following an argument of Crandall and Tartar [13, 14] we consider the initial value problem for data of arbitrary mass. Let $K_{0}$ be the maximum for the initial data. We choose $T$ and $x_{0}$ and construct a solution in the region

$$
\left\{(x, t): 0<t<T,\left|x-x_{0}\right|<c_{\max }(2 T-t)\right\} .
$$

Since the solution in this region is determined by the data for $\left|x-x_{0}\right| \leqq 2 c_{\max } T$, we may modify the data outside this interval to have compact support without affecting the solution in our region. This can be done so that $m$ and $K_{0}$ are not increased. We now consider the initial value problem for the modified data of compact support. Since the solution has compact support for each $t$, the entropy function

$$
\int_{-\infty}^{\infty} \sum_{j} v_{j} u_{j} \log u_{j} d x
$$


is defined and is nonincreasing (see Sect. I.D of [5] or Sect. 2.1 of [3]). Since $v \log v \geqq-1 / e$ for $v \geqq 0$, it follows that

$$
\int_{-\infty}^{\infty} \sum_{j} v_{j} u_{j} \log ^{+} u_{j} d x \leqq C\left(T, K_{0}\right)
$$

for $t \leqq T$, with $\log ^{+} v$ the positive part of $\log v$. It can be seen as a consequence (see the proof of Theorem 4 in [14]) that there exists a number $r$, depending only on $K_{0}$, $T$, so that

$$
\int_{I} \sum_{j} v_{j} u_{j}(x, t) d x \leqq 1 / 2 C_{0}
$$

for every interval $I$ of length $\leqq r$ with $I \times\{t\}$ in the region. The small mass existence result can now be applied to continue the solution for time steps of length $r / 3 c_{\text {max }}$. By varying $x_{0}$, we construct a $C^{1}$ solution of the original problem on $\mathbb{R} \times(0, T)$. Since the choice of $r$ can be made independent of $x_{0}$, the maximum of the solution is bounded by a number $G\left(T, K_{0}\right)$. By conservation of mass the solution is in $L^{1}$, and therefore $L^{p}, 1 \leqq p<\infty$, for each $t$. Since $T$ was arbitrary, we have such a solution for all time.

It remains to extend the existence theorem to the case of nonsmooth data. For $u_{0} \in L^{1} \cap L^{\infty}$ we can obtain the solution as the limit of smooth solutions by a process which will also be useful later. Let $\varphi(x)$ be a test function with

$$
\varphi \in C_{0}^{\infty}(\mathbb{R}), \quad \varphi \geqq 0, \quad \int_{-\infty}^{\infty} \varphi(x) d x=1,
$$

and let $\varphi_{k}(x)=k \varphi(k x)$. We replace $u_{0}$ by the convolution $u_{0}^{(k)}=u_{0} * \varphi_{k}$, and check that the solutions converge as $k \rightarrow \infty$ :

Lemma 3.1. With $u_{0} \in L^{1} \cap L^{\infty}$ and $T>0$ arbitrary, define $u_{0}^{(k)}=u_{0} * \varphi_{k}$ as above. Let $u^{(k)}$ be the solution of (1.1) with $u^{(k)}(0)=u_{0}^{(k)}$. Then the $u^{(k)}$ are uniformly bounded on $\mathbb{R} \times[0, T]$, and for $1 \leqq p<\infty, u^{(k)}$ converges in $C\left(0, T ; L^{p}\right)$ to a solution $u$ of (1.1) with $u(0)=u_{0}$. This solution is uniformly bounded on $\mathbb{R} \times[0, T]$ by $G\left(T,\left|u_{0}\right|_{L^{\infty}}\right)$.

Proof. Since $\left|u_{0}^{(k)}\right|_{L^{\infty}} \leqq\left|u_{0}\right|_{L^{\infty}}$, we have $\left|u^{(k)}(x, t)\right| \leqq G\left(T,\left|u_{0}\right|_{L^{\infty}}\right) \equiv K$, where $G$ is the function obtained in the above argument. Now if $v=u^{(k)}-u^{\left(k^{\prime}\right)}$, then

$$
v_{i, t}+c_{i} v_{i, x}=h_{i}(x, t), \quad\left|h_{i}(x, t)\right| \leqq C|v(x, t)|,
$$

where $C$ depends on $K$, but not on $k, k^{\prime}$. Define $f_{n} \in C^{1}(\mathbb{R})$ by $f_{n}(y)=|y|-1 /(2 n)$ for $|y| \geqq 1 / n, f_{n}(y)=n y^{2} / 2$ for $|y| \leqq 1 / n$. Then $0 \leqq f_{n}(y) \leqq|y|$, and $f_{n}(y) \rightarrow|y|$ as $n \rightarrow \infty$. Multiplying the above equation by $f_{n}^{\prime}\left(v_{i}\right)$, we have $f_{n}\left(v_{i}\right)_{t}+c_{i} f_{n}\left(v_{i}\right)_{x}=f_{n}^{\prime}\left(v_{i}\right) h(x, t)$. Integrating in $x$ and $t$, and then letting $n \rightarrow \infty$, we find

$$
\int_{-\infty}^{\infty}|v(x, t)| d x \leqq \int_{-\infty}^{\infty}\left|u_{0}^{(k)}-u_{0}^{\left(k^{\prime}\right)}\right| d x+C \int_{0}^{t} \int_{-\infty}^{\infty}|v(x, t)| d x d t
$$

Thus from Gronwall's inequality we have $\left|v(\cdot, t)_{L^{1}} \leqq e^{C t}\right| u_{0}^{(k)}-\left.u_{0}^{\left(k^{\prime}\right)}\right|_{L^{1}}$. Since $u_{0}^{(k)} \rightarrow u_{0}$ in $L^{1}(\mathbb{R})$, it follows that $u^{(k)}$ converges in $C\left(0, T ; L^{1}(\mathbb{R})\right)$ to a function $u(x, t)$. In fact, since the $u^{(k)}$ are uniformly bounded, the convergence takes place in $C\left(0, T ; L^{p}(\mathbb{R})\right)$, $1 \leqq p<\infty$. It is easily seen that the limit function is a distributional solution of the 
equation. Finally we check the uniform boundedness of $u$. For each $k, t, p$ we have

$$
\left|u^{(k)}(\cdot, t)\right|_{L^{p}} \leqq\left\{K^{p-1}\left|u^{(k)}(\cdot, t)\right|_{L^{1}}\right\}^{1 / p}=\left\{K^{p-1}\left|u_{0}^{(k)}\right|_{L^{1}}\right\}^{1 / p} \leqq K^{\frac{p-1}{p}}\left|u_{0}\right|_{L^{1}}^{1 / p}
$$

and thus $u(\cdot, t)$ has the same bound. Letting $p \rightarrow \infty$, we have $|u(\cdot, t)|_{L^{\infty}} \leqq K$.

To complete the proof of the Existence Theorem it remains only to verify the uniqueness property. For solutions in the prescribed regularity class we can easily check that for arbitrary $\varphi \in C_{0}^{\infty}(\mathbb{R})$ and $t>0$,

$$
\begin{aligned}
& \int_{-\infty}^{\infty} u_{i}\left(x+c_{i} t, t\right) \varphi(x) d x-\int_{-\infty}^{\infty} u_{0 i}(x, t) \varphi(x) d x \\
& =\int_{-\infty}^{\infty} \int_{0}^{t} F_{i}\left(x+c_{i} t^{\prime}, t^{\prime}\right) \varphi(x) d t^{\prime} d x .
\end{aligned}
$$

Therefore

$$
u_{i}\left(x+c_{i} t, t\right)-u_{0 i}(x)=\int_{0}^{t} F_{i}\left(x+c_{i} t^{\prime}, t^{\prime}\right) d t^{\prime}
$$

for almost all $(x, t)$. If there are two solutions with the same initial data and $y(t)$ is the $L^{\infty}$-norm of the difference at time $t$, we can obtain from this representation and the uniform boundedness an estimate

$$
y(t) \leqq c \int_{0}^{t} y\left(t^{\prime}\right) d t^{\prime}, \quad y(0)=0
$$

and this implies $y \equiv 0$.

\section{Asymptotics in $L^{1}$}

In this section we prove Theorem 1 . Here and subsequently we wll write $D_{i}$ for $D_{t}+c_{i} D_{x}$.

We begin by introducing functions $l_{i}(x, t)$ related to mass and momentum, analogous to those used in [1], so that $D_{i} l_{i}$ is a negative linear combination of the $u_{j}$. We assume for now that the solution under consideration is smooth. Define

$$
l^{(0)}(x, t)=\int_{-\infty}^{x} \sum_{j} v_{j} u_{j}\left(x^{\prime}, t\right) d x^{\prime} .
$$

Applying the divergence theorem to the mass conservation law (3.1) on the region $\left\{\left(x^{\prime}, t^{\prime}\right):-\infty<x^{\prime}<x, 0<t^{\prime}<i\right\}$, we get

$$
l^{(0)}(x, t)-l^{(0)}(x, 0)+\int_{0}^{t} \sum_{j} v_{j} c_{j} u_{j}\left(x, t^{\prime}\right) d t^{\prime}=0 .
$$

It is evident from the definition and from the above identity that

$$
D_{x} l^{(0)}=\sum v_{j} u_{j}, \quad D_{t} l^{(0)}=-\sum v_{j} c_{j} u_{j} .
$$

Thus

$$
D_{i} l^{(0)}=\sum_{j} v_{j}\left(c_{i}-c_{j}\right) u_{j}
$$


In just the same way we set

$$
l^{(1)}(x, t)=\int_{-\infty}^{x} \sum_{j} v_{j} c_{j} u_{j}\left(x^{\prime}, t\right) d x^{\prime},
$$

and use the conservation of momentum (3.2) to find

$$
D_{i} l^{(1)}=\sum_{j} v_{j} c_{j}\left(c_{i}-c_{j}\right) u_{j}
$$

Thus if $l_{i}=l^{(1)}-c_{i} l^{(0)}$, we have

$$
D_{i} l_{i}=-\sum_{j} v_{j}\left(c_{i}-c_{j}\right)^{2} u_{j} \leqq-\gamma_{i} \sum_{j \neq i} u_{j}
$$

for some constant $\gamma_{i}>0$, using (1.2).

We use the $l_{i}$ 's in the proof of the lemma below. The asymptotic description in $L^{1}$ will follow from this lemma.

Lemma 4.1. For each choice of $i, j, k$ so that $a_{i}^{j k} \neq 0$, we have

$$
\int_{0}^{\infty} \int_{-\infty}^{\infty} u_{j} u_{k} d x d t \leqq M
$$

where $M$ is a constant depending only on the mass $m$ and the coefficients in the equations.

Proof. We first assume the solution is smooth. Suppose for now that $i$ is chosen and $a_{i}^{i i}=0$. We write the $i^{\text {th }}$ equation as

$$
D_{i} u_{i}=\sum_{j, k \neq i} a_{i}^{j k} u_{j} u_{k}-\sum_{j \neq i} b_{j}^{i} u_{j} u_{i}
$$

with $b_{i}^{j}=-2 a_{i}^{i j}$, so that all coefficients in (4.1) are nonnegative. Let $l(x, t)=l_{i}(x, t) / \gamma_{i}$ with $l_{i}$ as above; then

$$
D_{i} l(x, t) \leqq-\sum_{j \neq i} u_{j}(x, t),
$$

and $|l(x, t)| \leqq C m \equiv \Lambda$ for all $(x, t)$. We will multiply (4.1) by $f^{\prime}(l)$, where $f$ is a smooth function to be determined. Assuming $f^{\prime}>0$ we have

$$
D_{i} f(l)=f^{\prime}(l) D_{i} l \leqq-\sum_{j \neq i} f^{\prime} u_{j},
$$

and writing $D_{i}\left(f u_{i}\right)=\left(D_{i} f\right) u_{i}+f\left(D_{i} u_{i}\right)$,

$$
D_{i}\left[f(l) u_{i}\right] \leqq-\sum_{j \neq i} f^{\prime} u_{j} u_{i}+\sum_{j, k \neq i} f a_{i}^{j k} u_{j} u_{k}-\sum_{j \neq i} f b_{i}^{j} u_{j} u_{i}
$$

We wish the coefficients of the terms on the right to be strictly less than they would be if $f$ were replaced by 1 . For this reason we will choose $f$ so that for $|l| \leqq \Lambda$, and some $\delta>0$,

$$
\delta \leqq f \leqq 1-\delta, \quad f^{\prime}>0, \quad f^{\prime}+b_{i}^{j} f \geqq b_{i}^{j}(1+\delta) .
$$

Let $\beta=\max b_{i}^{j}$, and define $f(l)=1+\delta-A e^{-\beta l}$ with $\delta, A$ to be determined. With $A>0, f$ will be increasing. We specify $f(\Lambda)=1-\delta$, or $A=2 \delta e^{\beta \Lambda}$. To ensure $f \geqq \delta$, we choose $\delta$ so that $f(-\Lambda)=\delta$. With $A$ as above, this means that $\delta=e^{-2 \beta \Lambda} / 2$. The 
first two conditions of (4.2) are now satisfied, and for the third we have

$$
f^{\prime}+b_{i}^{j} f=\left(\beta-b_{i}^{j}\right) A e^{-\beta l}+b_{i}^{j}(1+\delta) \geqq b_{i}^{j}(1+\delta) .
$$

As a consequence of (4.2) we can write

$$
D_{i}\left[f u_{i}\right] \leqq D_{i} u_{i}-\delta\left\{\sum_{j, k \neq i} a_{i}^{j k} u_{j} u_{k}+\sum_{j \neq i} b_{i}^{j} u_{j} u_{i}\right\}
$$

or

$$
D_{i}\left[f u_{i}\right] \leqq D_{i} u_{i}-\alpha \sum_{j, k}^{\prime} u_{j} u_{k}
$$

for some $\alpha>0$, where the prime denotes the sum over pairs $j, k$ with $a_{i}^{j k} \neq 0$. Next we multiply by $v_{i}$, add together $v_{i^{\prime}} D_{i^{\prime}} u_{i^{\prime}}$ for $i^{\prime} \neq i$, and use the conservation of mass relation (3.1) to obtain

$$
v_{i} D_{i}\left(f u_{i}\right)+\sum_{i^{\prime} \neq i} v_{i^{\prime}} D_{i^{\prime}} u_{i^{\prime}}+\alpha v_{i} \sum_{j, k}^{\prime} u_{j} u_{k} \leqq 0 .
$$

Finally, integrating over $x$ and from time 0 to $T$ gives

$$
\begin{gathered}
\int\left(v_{i} f u_{i}+\sum_{i^{\prime} \neq i} v_{i^{\prime}} u_{i^{\prime}}\right) d x+\alpha \sum_{j, k}^{\prime} \int_{0}^{T} \int u_{j} u_{k} d x d t \\
\leqq \int\left(v_{i} f u_{0 i}+\sum_{i^{\prime} \neq i} v_{i^{\prime}} u_{0 i^{\prime}}\right) d x \leqq m .
\end{gathered}
$$

The assertion follows since $T$ is arbitrary.

The above argument applies to each $i$ for which $a_{i}^{i i}=0$. If $a_{i}^{i i}<0$, then there is a sequence $i=i_{1}, \ldots, i_{r}$ as in assumption (1.9). Since $a_{i_{r}}^{i_{r} i_{r}}=0$, the above argument applies to the $i_{r}$-equation, and since a term $u_{i_{r-1}}^{2}$ appears in this equation, it is integrable,

$$
\int_{0}^{\infty} \int u_{i_{r-1}}^{2} d x d t \leqq m / \alpha
$$

We may argue as before for the $i_{r-1}$-equation, except that the term $u_{i_{r-1}}^{2}$ must be treated differently. Inequality (4.3) is replaced by

$$
D_{i_{r-1}}\left(f u_{i_{r-1}}\right) \leqq D_{i_{r-1}} u_{i_{r-1}}-\alpha \sum_{j, k}^{\prime} u_{j} u_{k}+C u_{i_{r-1}}^{2},
$$

and (4.4) by

$$
\int\left(v_{i_{r-1}} f u_{i_{r-1}}+\sum_{i^{\prime} \neq i_{r-1}} v_{i^{\prime}} u_{i^{\prime}}\right) d x+\alpha \sum_{j, k}^{\prime} \int_{0}^{T} \int u_{j} u_{k} d x d t \leqq m+C \int_{0}^{T} \int u_{i_{r-1}}^{2} d x d t
$$

for some constant $C$. Because of (4.5), we may conclude that the interaction terms in the $i_{r-1}$-equation are integrable. Now by assumption, the $i_{r-1}$-equation contains a term $u_{i_{r-2}}^{2}$, and we may repeat this argument for the $i_{r-2}$-equation. Continuing this process, we finally obtain the same conclusion for the $i$-equation.

If the solution is not smooth, we can approximate by solutions corresponding to mollified initial data as in Lemma 3.1. The modified data will converge in $L^{1}$ and be uniformly bounded in $L^{\infty}$. It follows from Lemma 3.1 that for any fixed $T$ the 
solutions converge in $C\left(0, T, L^{1} \cap L^{2}\right)$. The l's then converge uniformly. Consequently the estimates (4.4), (4.6), which hold for the smooth approximations, also hold for arbitrary solutions by passage to the limit. Once again, since $T$ was arbitrary, we may conclude that the interaction terms are integrable.

As observed in [14], the asymptotic description of solutions in $L^{1}(\mathbb{R})$ is a simple consequence of the above result:

Proof of Theorem 1. As in [14], we define $u_{i}^{*}(x, t)=u_{i}\left(x+c_{i} t, t\right)$ and $F_{i}^{*}(x, t)$ $=F_{i}\left(x+c_{i} t, t\right)$. Then $D_{t} u_{i}^{*}=F_{i}^{*}$. By the above lemma we can regard $F_{i}^{*}$ as an element of $L^{1}\left(0, \infty ; L^{1}(\mathbb{R})\right)$, and write

$$
u_{i}^{*}(\cdot, t)=u_{0 i}+\int_{0}^{t} F^{*}(\cdot, s) d s .
$$

Since the integral converges as $t \rightarrow \infty$, it is evident that $u_{i}^{*}(\cdot, t)$ approaches a limit $u_{i}^{\infty} \in L^{1}(\mathbb{R})$, or equivalently,

$$
\int\left|u_{i}(x, t)-u_{i}^{\infty}\left(x-c_{i} t, t\right)\right| d x \rightarrow 0 \quad \text { as } t \rightarrow \infty .
$$

It remains to show that if $a_{i}^{i i}=0$, then $u_{i}^{\infty}=0$, i.e., $u_{i}^{*}(\cdot, t) \rightarrow 0$ in $L^{1}(\mathbb{R})$. We know that $u_{i}^{*}(\cdot, t) \rightarrow u_{i}^{\infty}$ in $L^{1}$ and from the lemma that

$$
\int_{0}^{\infty} \int\left(u_{i}^{*}\right)^{2} d x d t<\infty
$$

In particular this holds on any finite interval $I$ on the $x$-axis. We can choose a sequence $t_{n} \rightarrow \infty$ so that

$$
\int_{I} u_{i}^{*}\left(x, t_{n}\right)^{2} d x \rightarrow 0 \quad \text { as } \quad n \rightarrow \infty
$$

and by the Cauchy-Schwarz inequality

$$
\int_{I} u_{i}^{*}\left(x, t_{n}\right) d x \rightarrow 0
$$

also. Since $u_{i}^{*} \rightarrow u_{i}^{\infty}$ in $L^{1}(I)$ and $u_{i}^{\infty} \geqq 0$, we must have $u_{i}^{\infty}=0$ on $I$. Since $I$ was arbitrary, $u_{i}^{\infty} \equiv 0$.

\section{Estimates and Asymptotics in Higher Norms}

In this section our main task is to estimate solutions in $L^{p}$, and thereby establish Theorem 2. We assume for now that the solutions are $C^{1}$.

We will estimate in space-time regions moving approximately with one of the speeds $c_{i}$. To localize we introduce numbers $r_{0}, \ldots, r_{n}, c_{1+}, \ldots, c_{n+}, c_{1-}, \ldots, c_{n-}$, so that

$$
c_{i}<r_{i}<c_{i+1}, \quad r_{i-1}<c_{i-}<c_{i}, \quad c_{i}<c_{i+}<r_{i},
$$

wherever defined. We partition the real line into intervals $I_{i}=\left(r_{i-1}, r_{i}\right]$, $i=2, \ldots, n-1$ and $I_{1}=\left(-\infty, r_{1}\right], I_{n}=\left(r_{n-1}, \infty\right)$. We will also use cut-off functions of $x / t$. For $1 \leqq i \leqq n$, let $\zeta_{i}^{-}, \zeta_{i}^{+}$be functions in $C^{\infty}(\mathbb{R})$ with values in $[0,1]$ so that $\zeta_{i}^{-}=1$ on $\left(-\infty, r_{i-1}\right], \zeta_{i}^{-}=0$ on $\left[c_{i-}, \infty\right), \zeta_{i}^{+}=0$ on $\left(-\infty, c_{i+}\right], \zeta_{i}^{+}=1$ on $\left[r_{i}, \infty\right)$. Thus $\zeta_{i}^{-}$is supported on speeds below $c_{i}$, and $\zeta_{i}^{+}$above. 
We will use integral quantities like the $l_{i}$ of the last section but modified with the cut-offs so that they are small for large $t$. Let $S$ be the set of indices $i$ so that $u_{i}^{2}$ terms occur in the equations, i.e. $a_{i}^{i i}<0$. Define first

$$
U_{k}(x, t)=\int_{-\infty}^{x} \zeta_{k}^{-}\left(x^{\prime} / t\right) u_{k}\left(x^{\prime}, t\right) d x^{\prime}-\int_{x}^{\infty} \zeta_{k}^{+}\left(x^{\prime} / t\right) u_{k}\left(x^{\prime}, t\right) d x^{\prime}
$$

for $k \notin S$ and

$$
U_{k}(x, t)=\int_{-\infty}^{x} u_{k}\left(x^{\prime}, t\right) d x^{\prime}, \quad k \in S .
$$

The analogue of $l_{j}$ will be

$$
h_{j}(x, t)=\sum_{k=1}^{n}\left(c_{k}-c_{j}\right) U_{k}(x, t) .
$$

Lemma 5.1. The $h_{j}$ have the following two properties: (i) $h_{j}(x, t) \rightarrow 0$ as $t \rightarrow \infty$, uniformly in $x$.

(ii) $D_{j} h_{j}=-\sum_{k \notin S}\left(c_{k}-c_{j}\right)^{2}\left(\zeta_{k}^{-}+\zeta_{k}^{+}\right) u_{k}-\sum_{k \in S}\left(c_{k}-c_{j}\right)^{2} u_{k}+g_{j}(x, t)$ for certain functions $g_{j}(x, t)$. There are functions $G_{j}(t)$, and a constant $M_{1}$ depending only on $m$, so that

$$
\left|g_{j}(x, t)\right| \leqq G_{j}(t), \quad \int_{0}^{\infty} G_{j}(t) d t \leqq M_{1} .
$$

Proof. For $k \in S, u_{k}(\cdot, t) \rightarrow 0$ in $L^{1}(\mathbb{R})$ and thus $U_{k}(x, t) \rightarrow 0$ uniformly in $x$. For $k \notin S$,

$$
U_{k}(x, t) \leqq \int_{-\infty}^{c_{k}-t} u_{k} d x^{\prime}+\int_{c_{k}+t}^{\infty} u_{k} d x^{\prime}
$$

Now for large $t, u_{k}(x, t)$ is close in $L^{1}(\mathbb{R})$ to $u_{k}^{\infty}\left(x-c_{k} t\right)$, and the first integral is therefore close to

$$
\int_{-\infty}^{c_{k-1} t} u_{k}^{\infty}\left(x-c_{k} t\right) d x=\int_{-\infty}^{\left(c_{k}-c_{k}\right) t} u_{k}^{\infty}(x) d x .
$$

Since $c_{k-}<c_{k}$, the last integral goes to zero at $t \rightarrow \infty$. The second term can be estimated in the same way, showing that $U_{k} \rightarrow 0$ uniformly in $t$. Conclusion (i) follows.

Let $U_{k}^{-}$be the first term in the definition of $U_{k}$ for $k \notin S$. Writing the equation for $u_{k}$ as $D_{k} u_{k}=F_{k}$, we have

$$
D_{k}\left(\zeta_{k}^{-} u_{k}\right)=\zeta_{k}^{-} F_{k}+\left(D_{k} \zeta_{k}^{-}\right) u_{k}
$$

Integrating over the region $\left\{\left(x^{\prime}, t^{\prime}\right): x^{\prime}<x, 0<t^{\prime}<t\right\}$ gives

$$
U_{k}^{-}(x, t)+\int_{0}^{t} c_{k}\left(\zeta_{k}^{-} u_{k}\right)\left(x, t^{\prime}\right) d t^{\prime}-U_{k}^{-}(x, 0)=\int_{0}^{t} \int_{-\infty}^{x}\left[\zeta_{k}^{-} F_{k}+\left(D_{k} \zeta_{k}^{-}\right) u_{k}\right] d x^{\prime} d t^{\prime} .
$$

Evidently $D_{x} U_{k}^{-}=\zeta_{k}^{-} u_{k}$, and

$$
D_{t} U_{k}^{-}=-c_{k} \zeta_{k}^{-} u_{k}+\int_{-\infty}^{x}\left[\zeta_{k}^{-} F_{k}+\left(D_{k} \zeta_{k}^{-}\right) u_{k}\right] d x^{\prime} .
$$


Now $D_{k} \zeta_{k}^{-}=\left(\zeta_{k}^{-}\right)^{\prime}(x / t) \cdot\left(c_{k} t-x\right) / t^{2}$, which is bounded by $C / t$. Thus the last term in (5.1) is bounded by a constant times

$$
\int_{-\infty}^{\infty}\left|F_{k}(x, t)\right| d x+\frac{1}{t} \int_{\alpha t}^{\beta t} u_{k}(x, t) d x,
$$

where $\alpha=r_{k-1}, \beta=c_{k-}$. The first of these is integrable in $t$ by Lemma 4.1. We need to estimate

$$
\int_{0}^{\infty} \int_{\alpha t}^{\beta t} \frac{1}{t} u_{k}(x, t) d x d t
$$

Let $x=(1-\theta) \alpha t+\theta \beta t$. We change the variables to $(\theta, t)$. The Jacobian is $(\beta-\alpha) t$, and the integral becomes

$$
(\beta-\alpha) \int_{0}^{1} \int_{0}^{\infty} u_{k}((1-\theta) \alpha t+\theta \beta t, t) d t d \theta
$$

It will be enough to estimate the $t$-integral with $\theta$ fixed. With $c^{*}=(1-\theta) \alpha+\theta \beta$, $u_{k}^{*}(x, t)=u_{k}\left(x+c^{*} t, t\right)$, and the same for $F_{k}^{*}$, we have $u_{k, t}^{*}+\left(c_{k}-c^{*}\right) u_{k, x}^{*}=F_{k}^{*}$ and $c_{k}>c^{*}$. Integrating over $\left\{\left(x^{\prime}, t^{\prime}\right): x^{\prime}<0,0<t^{\prime}<t\right\}$, we find

$$
\int_{-\infty}^{0} u_{k}^{*}(x, t) d x+\left(c_{k}-c^{*}\right) \int_{0}^{t} u_{k}^{*}\left(0, t^{\prime}\right) d t^{\prime}=\int_{-\infty}^{0} u_{k}^{*}(x, 0) d x+\int_{0}^{t} \int_{-\infty}^{0} F_{k}^{*} d x d t^{\prime} .
$$

As $t \rightarrow \infty$, the integral in the second term approaches our integral above, while the other terms are bounded by constants depending on $m$. It follows that the last term in (5.1) is integrable in $t$. We have essentially verified conclusion (ii) for the $\zeta_{k}^{-}$ terms, $k \notin S$. The $\zeta_{k}^{+}$terms may be treated in the same way, and the terms $k \in S$ are of course simpler.

We now begin the estimates in $L^{p}$. We choose $i$ and estimate for $x / t \in I_{i}$, assuming at first that $i \notin S$. With the $j^{\text {th }}$ equation in the form $D_{j} u_{j}=F_{j}$, we introduce a weight function $\mu_{j}(x, t)$ to be chosen and write

$$
D_{j}\left(\mu_{j} u_{j}^{p}\right)=\left(D_{j} \mu_{j}\right) u_{j}^{p}+p \mu_{j} u_{j}^{p-1} F_{j} .
$$

We define

$$
\mu_{j}(x, t)=\exp \left\{\gamma p\left(h_{j}(x, t)+\eta\right)\right\},
$$

with $\gamma$ and $\eta$ constants to be chosen later. Thus

$$
D_{j} \mu=\gamma p \mu_{j}\left(D_{j} h_{j}\right) \text {. }
$$

In Lemma 5.1 we found an expression for $D_{j} h_{j}$. For $k \neq i$, one of $\zeta_{k}^{-}, \zeta_{k}^{+}$is 1 and the other 0 for $x / t \in I_{i}$. Therefore

$$
D_{j} \mu_{j} \leqq-p \delta \gamma \mu_{j} \sum_{k \neq i, j} u_{k}+p \gamma G_{j} \mu_{j}
$$

for some $\delta>0, x / t \in I_{i}$, using (1.2).

The last term in (5.2) is

$$
p \mu_{j}\left\{\sum_{k, l \neq j} a_{j}^{k l} u_{k} u_{l} u_{j}^{p-1}-\sum_{k} b_{j}^{k} u_{k} u_{j}^{p}\right\},
$$


where $b_{j}^{k}=-2 a_{j}^{j k}$ for $k \neq j, b_{j}^{j}=-a_{j}^{j j}$. We estimate the growth terms using Young's inequality:

$$
\mu_{j} u_{l} u_{j}^{p-1} \leqq \frac{1}{p} u_{l}^{p}+\frac{p-1}{p}\left(\mu_{j}\right)^{\frac{p}{p-1}} u_{j}^{p},
$$

and thus

$$
p \mu_{j} u_{k} u_{l} u_{j}^{p-1} \leqq u_{k} u_{l}^{p}+p\left(\mu_{j}\right)^{\frac{p}{p-1}} u_{k} u_{j}^{p}
$$

Since we have assumed that $i \notin S$, one of $k, l$ is not $i$; take $k \neq i$.

Combining inequalities, we can write

$$
\begin{aligned}
D_{j}\left(\mu_{j} u_{j}^{p}\right) \leqq & -p \gamma \delta \mu_{j} \sum_{k \neq i, j} u_{k} u_{j}^{p}+\sum_{k \neq i, j} \sum_{l \neq j} p a_{j}^{k l}\left(\mu_{j}\right)^{\frac{p}{p-1}} u_{k} u_{j}^{p} \\
& +\sum_{k \neq i, j} \sum_{l \neq j} a_{j}^{k l} u_{k} u_{l}^{p}-\sum_{k} p b_{j}^{k} \mu_{j} u_{k} u_{j}^{p}+p \gamma G_{j} \mu_{j} u_{j}^{p} .
\end{aligned}
$$

We will use the first term on the right to dominate the second and, after summing in $j$, the first and fourth to control the third. For the first case we will choose the constants in $\mu_{j}$ so that for each $k \neq i, j$,

$$
\sum_{l \neq j} a_{j}^{k l}\left(\mu_{j}\right)^{\frac{p}{p-1}} \leqq \gamma \delta \mu_{j} / 2
$$

It will be sufficient to replace this by

$$
\left(\mu_{j}\right)^{\frac{1}{p-1}} \leqq \gamma \delta / A
$$

where $A / 2$ is an upper bound for the coefficient sum, or for $p \geqq 2$ by

$$
\exp \left(2 \gamma\left(h_{j}+\eta\right)\right) \leqq \gamma \delta / A
$$

To satisfy this condition, we first choose $\gamma=e A / \delta$. Now according to Lemma 5.1, there is a $T$ sufficiently large so that $\left|h_{j}(x, t)\right| \leqq 1 / 4 \gamma$ for $t>T$. We take $\eta=1 / 4 \gamma$; then for $t>T(5.6)$, and therefore (5.5), is satisfied. In addition we have ensured that $\mu_{j} \geqq 1$.

Next we bound the third term in (5.4). Suppose $a_{j}^{k l}>0$ with $k \neq i, j$ and $l \neq j$. We consider two cases: (i) $k \neq l$, (ii) $k=l$. In case (i), since $k \neq i$, the version of (5.4) with $j$ replaced by $l$ contains a term $-p \gamma \delta \mu_{l} u_{k} u_{l}^{p}$. Because $\mu_{l} \geqq 1$ and because of the factor of $p$, this term dominates the term $a_{j}^{k l} u_{k} u_{l}^{p}$ from the $j$-equation for sufficiently large $p$; in fact it dominates any number of such terms. In case (ii), we have $a_{j}^{l l}>0$ with $l \neq i$. By the conservation of mass relation (1.6), there is a negative $u_{l}^{2}$ term in the $l$-equation. Thus $b_{l}^{l}>0$, and (5.4) with $j$ replaced by $l$ contains a term $-p b_{l}^{l} \mu_{l} u_{l}^{p+1}$. This dominates the growth term $a_{j}^{l l} u_{l}^{p+1}$ for the same reasons as in case (i). Finally we multiply (5.4) by $v_{j}$, sum over $j$, and combine terms using the results of the last two paragraphs to obtain for $t \geqq T, x / t \in I_{i}$, and sufficiently large $p$,

$$
\sum_{j} D_{j}\left(v_{j} \mu_{j} u_{j}^{p}\right) \leqq p \gamma G(t) \sum_{j} v_{j} \mu_{j} u_{j}^{p}
$$

Here $G(t)=\max G_{j}(t)$.

We check that (5.7) holds also in the case $i \in S$. Now (5.5) can be strengthened to include the term $k=i$, assuming $j \neq i$. The first three terms on the right in (5.4) now 
have $k \neq j$ rather than $k \neq i, j$. Otherwise the argument proceeds as before. Thus (5.7) holds for all $(x, t)$ with $t \geqq T$.

Now let

$$
y(t)=\int_{-\infty}^{\infty} \sum_{j} v_{j} \mu_{j} u_{j}^{p}(x, t) d x .
$$

Integrating (5.7) over $x$, we have

$$
y^{\prime}(t) \leqq p \gamma G(t) y(t)
$$

and integrating in $t$,

$$
y(t) \leqq y(T) \exp \left(p \gamma \int_{T}^{\infty} G(t) d t\right) \leqq y(T) e^{p \gamma M_{1}}
$$

for $t \geqq T$. From the definition of $h_{j}$ and $\mu_{j}, \mu_{j}(x, t) \leqq C_{1}^{p} e^{p C_{2} m}$ with some constants $C_{1}, C_{2}$. Let $K_{0}$ be the maximum of the $u_{j}$ for $(\{x, t): 0 \leqq t \leqq T\}$; then

$$
y(T) \leqq C_{0} C_{1}^{p} e^{p C_{2} m} K_{0}^{p-1} m
$$

and

$$
y(t) \leqq C_{0} C_{1}^{p} e^{p M_{2}} K_{0}^{p-1} m, \quad M_{2}=C_{2} m+\gamma M_{1} .
$$

Since $\mu_{j} \geqq 1,\left|u_{j}(\cdot, t)\right|_{L^{p}} \leqq y(t)^{1 / p}$, and

$$
\left|u_{j}(\cdot, t)\right|_{L^{p}} \leqq C_{0}^{1 / p} C_{1} e^{M_{2}} \frac{p-1}{K_{0}^{p}} m^{1 / p}, \quad t \geqq T .
$$

Finally, we let $p \rightarrow \infty$. The $L^{p}$ norm converges to the $L^{\infty}$ norm, and we obtain

$$
\left|u_{j}(x, t)\right| \leqq C_{1} K_{0} e^{M_{2}}, \quad t \geqq T \text {. }
$$

Thus the $u_{j}$ are uniformly bounded for all $t \geqq 0$.

We have proved uniform boundedness for $C^{1}$ solutions. For initial data in $L^{1} \cap L^{\infty}$ we can modify the above argument to obtain the same conclusion. We approximate by smooth solutions as in Sect. 3. Repeating the above argument for the approximants, but without combining the first and second terms in (5.4), we can establish the following estimate for all $T, t$ and sufficiently large $p$ :

$$
\int_{-\infty}^{\infty} \sum_{j} v_{j} \mu_{j} u_{j}^{p} d x \leqq \int_{T}^{t} \int_{-\infty}^{\infty} R(x, t) d x d t+\int_{T}^{t} p \gamma G(t) \int_{-\infty}^{\infty} \sum_{j} v_{j} \mu_{j} u_{j}^{p} d x,
$$

with

$$
R(x, t)=-\frac{1}{2 p} \gamma \delta \sum_{j} \sum_{k}^{\prime} \mu_{j} u_{k} u_{j}^{p}+\sum_{j} \sum_{k, l}^{\prime} p a_{j}^{k l}\left(\mu_{j}\right)^{\frac{p}{p-1}} u_{k} u_{j}^{p},
$$

where the primes on the sums denote the previous exceptions. There is no difficulty in passing to the limit in this expression since the $h_{j}$ and $\mu_{j}$ converge uniformly. After doing so, we can show as before that $R \leqq 0$ for sufficiently large $t$, and we may use Gronwall's inequality to estimate as earlier.

We have already proved in Sect. 4 that $u_{j}^{*}(\cdot, t)=u_{j}\left(\cdot+c_{j} t, t\right)$ converges in $L^{1}$ to $u_{j}^{\infty}$. Since $u_{j}$ is uniformly bounded, it also converges in $L^{p}$ for $1 \leqq p<\infty$. The $L^{p}$ 
norms of the $u_{j}^{*}$ are bounded uniformly in $t$ as in (5.8), and therefore $u_{j}^{\infty}$ satisfies the same estimate in $L^{p}$. Letting $p \rightarrow \infty$, we find that $u_{j}^{\infty}$ is uniformly bounded in $x$ as in (5.9). This completes the proof of Theorem 2.

\section{Uniform Asymptotics}

In this section we prove Theorem 3. We assume throughout that $u_{0} \in L^{1} \cap L^{\infty}$ and $u_{0 j}(x) \rightarrow 0$ as $x \rightarrow \pm \infty$. $K$ will denote an upper bound for the solution, which is known to exist from the results of Sect. 5. We make use of ideas of Tartar [14].

We can express the solution of $D_{j} u_{j}=F_{j}$, integrating along characteristics, as

$$
u_{j}\left(\xi+c_{j} t, t\right)=u_{0 j}(\xi)+\int_{0}^{t} F_{j}\left(\xi+c_{j} s, s\right) d s .
$$

Since $F_{j} \in L^{1}(\mathbb{R} \times(0, \infty))$, the integral on the right remains bounded as $t \rightarrow \infty$ for almost all $\xi$, and therefore this expression is valid for all $t$ for a.a. $\xi$. (The exceptional set of $\xi$ is independent of $t$.) Letting $x=\xi+c_{j} t$, we have

$$
u_{j}(x, t)=u_{0 j}\left(x-c_{j} t\right)+\int_{0}^{t} F_{j}\left(x-c_{j} t+c_{j} s, s\right) d s,
$$

so that $u_{j}(x, t) \leqq g_{j}\left(x-c_{j} t\right)$, where as in [14] we define

$$
g_{j}(\xi)=u_{0 j}(\xi, t)+\int_{0}^{\infty}\left|F_{j}\left(\xi+c_{j} s, s\right)\right| d s .
$$

Then $g_{j} \in L^{1}(\mathbb{R})$ since $u_{0 j} \in L^{1}(\mathbb{R}), F_{j} \in L^{1}(\mathbb{R} \times(0, \infty))$.

Lemma 6.1. For each $\varepsilon>0$ there exists $A>0$ so that for all $t,\left|u_{i}(x, t)\right| \leqq \varepsilon$ for a.a. $x$ with $\left|x-c_{i} t\right| \geqq A$.

Proof. We fix $i$ and assume for simplicity that $c_{i}=0$. This is no restriction, since we can rewrite the equations with $x-c_{i} t$ as a new $x$-variable; the speed $c_{j}$ is then replaced by $c_{j}-c_{i}$. Integrating in $t$ and regarding $t$ as fixed, we have

$$
u_{i}(x, t) \leqq u_{0 i}(x)+\int_{0}^{t} \sum_{j, k \neq i} a_{i}^{j k} u_{j}\left(x, t^{\prime}\right) u_{k}\left(x, t^{\prime}\right) d t^{\prime} .
$$

By assumption $u_{0 i}(x)$ is small for large $|x|$. We will estimate the integral terms first for $j \neq k$. Now for every $x, u_{j}\left(x, t^{\prime}\right) \leqq g_{j}\left(x-c_{j} t^{\prime}\right)$ for a.a. $t^{\prime} \leqq t$; the exceptional set of $\left(x, t^{\prime}\right)$ for this estimate intersects a vertical line only in a set of measure zero since $j \neq i$ and $c_{j} \neq 0, c_{i}=0$. Also $u_{j} \leqq K$ for a.a. $\left(x, t^{\prime}\right)$, and therefore for a.a. $x$ this inequality holds for a.a. $t^{\prime}$. Then $u_{j}\left(x, t^{\prime}\right) \leqq \inf \left\{g_{j}\left(x-c_{j} t^{\prime}\right), K\right\}$, or equivalently, $u_{j}\left(x, t^{\prime}\right) \leqq h_{j}\left(t^{\prime}-x / c_{j}\right)$, where $h_{j}(s)=\inf \left\{g_{j}\left(-c_{j} s\right), K\right\}$. Since $g_{j} \in L^{1}(\mathbb{R}), h_{j} \in L^{2}(\mathbb{R})$. With the same considerations applied to $u_{k}$, the $j, k$-term in (6.2) is now estimated for a.a. $x$ by

$$
C \int_{-\infty}^{\infty} h_{j}\left(t^{\prime}-x / c_{j}\right) h_{k}\left(t^{\prime}-x / c_{k}\right) d t^{\prime}
$$

We claim that for $j \neq k$ this integral is small for sufficiently large $|x|$. Since $c_{j} \neq c_{k}$, this would be obvious if $h_{j}, h_{k}$ had compact support. If $h_{j}$ and $h_{k}$ are approximated 
in $L^{2}$ by functions of compact support with error $\delta$, then the error in the above integral is $0(\delta)$, uniformly in $x$ by the Cauchy-Schwarz inequality, and thus the claim holds in general. We conclude that for $j \neq k$, the $j, k$-term in (6.2) is uniformly small for all $(x, t)$ with $|x|$ sufficiently large, excepting for each $t$ a set of $x$ of measure zero.

To complete the proof of the lemma, it remains to derive a similar conclusion for the case $j=k$. If a term $u_{j}^{2}$ appears, we know that $u_{j}(\cdot, t) \rightarrow 0$ in $L^{1}(\mathbb{R})$ as $t \rightarrow \infty$. First we claim that for every $t$, for a.a. $x$,

$$
\begin{aligned}
c_{j} \int_{0}^{t} u_{j}\left(x, t^{\prime}\right) d t^{\prime}= & \int_{x}^{\infty} u_{j}\left(x^{\prime}, t\right) d x^{\prime}-\int_{x}^{\infty} u_{0 j}\left(x^{\prime}\right) d x^{\prime} \\
& -\int_{0}^{t} \int_{x}^{\infty} F_{j}\left(x^{\prime}, t^{\prime}\right) d x^{\prime} d t^{\prime} .
\end{aligned}
$$

If $u$ were smooth, this would follow from the divergence theorem. In general, we can approximate $u$ by smooth solutions as in Lemma 3.1. As we pass to the limit. each side of the equation converges in $L_{\text {loc }}^{1}(\mathbb{R})$, and therefore the two limits are equal for a.a. $x$. This verifies the claim. Now since $F_{j} \in L^{1}(\mathbb{R} \times(0, \infty))$ we can choose $A$ so that the last term in (6.3) is $\leqq \delta$ in magnitude for $x \geqq A$, where $\delta$ is an arbitrary small number. Similarly, the next to last term is also $\leqq \delta$ for large enough $x$. Since $u_{j}(\cdot, t) \rightarrow 0$ in $L^{1}(\mathbb{R})$, the first term on the right is $\leqq \delta$ for large enough $t$. Therefore the $t^{\prime}$-integral is bounded by $3 \delta /\left|c_{j}\right|$. For $x$ near $-\infty$, we can argue in a similar way, this time using the identity

$$
\begin{aligned}
c_{j} \int_{0}^{t} u_{j}\left(x, t^{\prime}\right) d t^{\prime}= & -\int_{-\infty}^{x} u_{j}\left(x^{\prime}, t\right) d x^{\prime}+\int_{-\infty}^{x} u_{0 j}\left(x^{\prime}\right) d x^{\prime} \\
& +\int_{0}^{t} \int_{-\infty}^{x} F_{j}\left(x^{\prime}, t^{\prime}\right) d x^{\prime} d t^{\prime} .
\end{aligned}
$$

We choose $A$ large enough so that the second and third terms on the right are bounded by $\delta$ for $x<-A$ and arrive at the same estimate for the $t^{\prime}$-integral. Finally, we note again that for each $t$, for a.a. $x$ we have $u_{j}\left(x, t^{\prime}\right) \leqq K$ for a.a. $t^{\prime} \leqq t$. Therefore

$$
\int_{0}^{t} u_{j}^{2}\left(x, t^{\prime}\right) d t^{\prime} \leqq K \int_{0}^{t} u_{j}\left(x, t^{\prime}\right) d t^{\prime} \leqq 3 K \delta /\left|c_{j}\right|
$$

for a.a. $x$ with $|x| \geqq A$. We have now shown that all the terms in (6.2) are small for large $|x|$, and the lemma is proved.

We are now ready to prove the uniform convergence of $u_{i}$. We assume first that $i \notin S$, and again take $c_{i}=0$ for convenience. We will show that for each $\varepsilon$ there exists $T$ sufficiently large so that for $t \geqq T$,

$$
\left|u_{i}(x, t)-u_{i}(x, T)\right| \leqq \varepsilon \text { for a.a. } x \text {. }
$$

From the lemma we may choose $A=A(\varepsilon)$ so that $u_{i}(x, t) \leqq \varepsilon / 2$ for $|x| \geqq A$ uniformly in $t$, and thus it suffices to verify (6.4) only for $|x| \leqq A$.

For each $T, t$, and a.a.x, we can write

$$
\left|u_{i}(x, t)-u_{i}(x, T)\right| \leqq \sum_{j, k}\left|a_{i}^{j k}\right| \int_{T}^{t} u_{j} u_{k} d t^{\prime},
$$


where for each term on the right, one of $j, k$ is $\neq i$, since $i \notin S$. Again for a.a. $x$ we have $u_{j}\left(x, t^{\prime}\right) \leqq g_{j}\left(x-c_{j} t^{\prime}\right)$ and $u_{k}\left(x, t^{\prime}\right) \leqq K$ for a.a. $t^{\prime}$; we suppose $j \neq i$ here. Then

$$
\int_{T}^{t} u_{j} u_{k} d t^{\prime} \leqq K \int_{T}^{\infty} g_{j}\left(x-c_{j} t^{\prime}\right) d t^{\prime}
$$

The last integral is $\pm c_{j}^{-1}$ times

$$
\int_{-\infty}^{x-c_{j} T} g_{j}(s) d s \text { or } \int_{x-c_{j} T}^{\infty} g_{j}(s) d s
$$

depending on whether $c_{j}>0$ or $c_{j}<0$. In either case the integral goes to zero as $T \rightarrow \infty$, uniformly for $|x| \geqq A$. This establishes (6.4) for $i \notin S$.

We now consider the case $i \in S$, again taking $c_{i}=0$. We begin by writing for $t_{2}>t_{1}$

$$
u_{i}\left(x, t_{2}\right)-u_{i}\left(x, t_{1}\right)+b \int_{t_{1}}^{t_{2}} u_{i}\left(x, t^{\prime}\right)^{2} d t^{\prime} \leqq \sum_{j, k \neq i} a_{i}^{j k} \int_{t_{1}}^{t_{2}} u_{j} u_{k} d t^{\prime} \equiv R\left(x, t_{1}, t_{2}\right)
$$

with $b=-a_{i}^{i i}>0$; in view of our earlier remarks this holds for all $\left(x, t_{1}, t_{2}\right)$ except for $x$ in a set of measure zero. Estimating the terms on the right just as above, we may find $T_{1}$ sufficiently large so that $R\left(x, T_{1}, \infty\right) \leqq \varepsilon / 2$ for $|x| \leqq A, x$ outside a set of measure zero. Then for $T_{2}>T_{1}$, bounding $u_{i}\left(x, T_{1}\right)$ by $K$,

$$
b \int_{T_{1}}^{T_{2}} u_{i}^{2} d t^{\prime} \leqq K+\varepsilon / 2 .
$$

If $u_{i} \geqq \varepsilon / 2$ on $\left[T_{1}, T_{2}\right]$, then the left-hand side is larger than $b \varepsilon^{2}\left(T_{2}-T_{1}\right) / 4$; for large enough $T_{2}$, this would contradict the above inequality. Therefore for $T_{2}=T_{1}$ $+4(K+1) b^{-1} \varepsilon^{-2}$, there is some $t_{1}$ depending on $x$ with $T_{1} \leqq t_{1} \leqq T_{2}$ so that $u_{i}\left(x, t_{1}\right) \leqq \varepsilon / 2$. But then from $(6.5), u_{i}(x, t) \leqq \varepsilon$ for all $t \geqq t_{1}$, and thus for all $t \geqq T_{2}$. Thus we have shown that, given $\varepsilon$ and $A$, there exists $T_{2}$ so that for $t \geqq T$,

$$
\left|u_{i}(x, t)\right| \leqq \varepsilon \text { for a.a. } x \text { with }|x| \leqq A .
$$

Together with Lemma 6.1 and our earlier conclusion for $i \notin S$, this completes the proof that the solution converges uniformly as $t \rightarrow \infty$.

Finally, since $u_{i}\left(x_{i}+c_{i} t, t\right)$ is uniformly close to $u_{i}^{\infty}(x)$ for $t$ large, and since by Lemma $6.1, u_{i}\left(x+c_{i} t, t\right)$ is uniformly small for large $|x|$, it follows that $u_{i}^{\infty}(x)$ is small for large $|x|$; i.e., $u_{i}^{\infty}(x) \rightarrow 0$ as $x \rightarrow \pm \infty$. If the $u_{0 i}$ are $C^{1}$, the solution is $C^{1}$ in spacetime, and the uniform convergence as $t \rightarrow \infty$ implies that $u_{i}^{\infty}$ is continuous.

\section{References}

1. Beale, J.T.: Large-time behavior of the Broadwell model of a discrete velocity gas. Commun. Math. Phys. 102, 217-235 (1985)

2. Cabannes, H.: Solution globale du probleme de Cauchy en théorie cinétique discrète. J. Méc. 17, 1-22 (1978)

3. Cabannes, H.: The discrete Boltzmann equation (theory and applications), lecture notes, University of California, Berkeley (1980)

4. Cabannes, H.: Comportement asymptotique des solutions de l'équation de Boltzmann discrète. C.R. Acad. Sci. Paris 302, S. 1, 249-253 (1986) 
5. Gatignol, R.: Théorie cinétique des gaz à répartition discrète de vitesses. Lecture Notes in Physics, Vol. 36. Berlin, Heidelberg, New York: Springer 1975

6. Godunov, S.K., Sultangazin, U.M.: On discrete models of the kinetic Boltzmann equation. Russ. Math. Surveys 26, No. 3, 1-56 (1971)

7. Hamdache, K.: Existence globale et comportement asymptotique pour l'équation de Boltzmann à répartition discrète des vitesses. J. Méc. Théor. Appl. 3, 761-785 (1984)

8. Hamdache, K.: On the discrete velocity models of the Boltzmann equation. Preprint

9. Illner, R.: Global existence results for discrete velocity models of the Boltzmann equation in several dimensions. J. Méc. Théor. Appl. 1, 611-622 (1982)

10. Kaniel, S., Shinbrot, M.: The Boltzmann equation, II: Some discrete velocity models. J. Méc. 19, 581-593 (1980)

11. Kawashima, S.: Global solution of the initial value problem for a discrete velocity model. Proc. Jpn. Acad. 57, 19-24 (1981)

12. Nishida, T., Mimura, M.: On the Broadwell's model for a simple discrete velocity gas. Proc. Jpn. Acad. 50, 812-817 (1974)

13. Tartar, L.: Existence globale pom un système hyperbolique semilinéarie de la théorie cinétique des gaz. Séminaire Goulaouic-Schwarz, No. 1 (1975/1976)

14. Tartar, L.: Some existence theorems for semilinear hyperbolic systems in one space variable, MRC Technical Summary Report, University of Wisconsin (1980)

Communicated by J. L. Lebowitz

Received January 28, 1986; in revised form April 24, 1986 